\title{
Classical model of bosons and fermions
}

\author{
G. Kaniadakis , P. Quarati \\ Dipartimento di Fisica del Politecnico, Corso Duca degli Abruzzi 2410129 Torino, Italy \\ Consorzio Interuniversitario Nazionale di Fisica della Materia, Unitá del Politecnico di Torino \\ Istituto Nazionale di Fisica Nucleare, Sezioni di Torino e di Cagliari
}

(March 10, 1994)

\section{IN COURSE OF PUBLICATION ON PHYS.REV. E}

\begin{abstract}
In a previous work we have derived a non-linear 1-dimensional kinetic equation for the distribution function of particles obeying an exclusion principle (EP). In the present work, on the same grounds, we extend this kinetics to D-dimensional continuous or discrete space, in order to study the distribution function of particles obeying a generalized exclusion-inclusion Pauli principle (EIP). This exclusion or inclusion principle is introduced into the classical transition rates by means of an inhibition or an enhancement factor, which contains a parameter $\kappa$, whose values range between -1 and +1 and can balance the effect of the full or partial validity of EIP. After deriving the kinetic equation we obtain a general expression of the stationary distribution function, depending on the value we give to the parameter $\kappa$. When we limit ourselves to brownian particles, we derive exactly for $\kappa=-1$ the Fermi-Dirac (FD) distribution, for $\kappa=0$ the Maxwell-Boltzmann (MB) distribution and for $\kappa=1$ the Bose-Einstein (BE) distribution. When $\kappa$ assumes an intermediate value, except zero, between the extreme values -1 and +1 , we obtain statistical distributions different from the FD and $\mathrm{BE}$ ones. We attribute to the parameter $\kappa$ the meaning of degree of indistinguishability of identical particles or of degree of antisymmetrisation or symmetrisation of the wave
\end{abstract}


function of the particle system.

02.50.Ga, 05.30.Fk, 05.40.+j, 05.20.Dd 


\section{INTRODUCTION}

In quantum physics, if there is appreciable overlapping of the wave functions of two identical particles in a system, non classical effects arise from the indistinguishability of identical particles. In this work we shall see how quantum considerations, particularly indistinguishability of identical particles, force significant changes in the classical procedures; particularly we shall derive and solve a non-linear kinetic equation describing the evolution of a system of indistinguishable particles; the quantum effects are contained in the transition probability rates and therefore, as a consequence, in the kinetic equation.

It is well known that, at thermal equilibrium, the presence of one classical and distinguishable particle in some particular energy state in no way inhibits or enhances the chance that another identical and distinguishable particle will be in that state. On the other hand, the effect of indistinguishability of quantum particles is that the presence of one in a particular quantum state very definitely influences the chance that another identical particle will be in that state.

For quantum indistinguishable particles, bosons or fermions, we can remark that the relations between the quantum transition rates and the classical transition rates are well known, at thermal equilibrium, where the detailed balancing postulate is the starting point for deriving classical and quantum Fermi-Dirac (FD) and Bose-Einstein (BE) distribution functions. If there are already $n$ bosons in a quantum state, the probability of one more joining them is larger by an enhancement factor of $1+n$ than it would be if there were no quantum mechanical indistinguishability requirements. If the occupation number of a system of fermions in a quantum state is $n$, the probability of one more fermion joining them is smaller by an inhibition factor of $1-n$ than it would be if there were no quantum mechanical indistinguishability requirements. If $n=0$ there is no inhibition of the probability for the first fermion entering the state. But for $n=1$ a second fermion is strictly inhibited from entering the same state.

Let us clarify the above arguments with this example. If we consider only two quantum 
states 1 and 2 and the two bosonic transition rates $r_{12}^{b}, r_{21}^{b}$ between the two states, we can obtain their values simply multiplying the classical rates $r_{12}^{c}, r_{21}^{c}$ by the enhancement factor $1+n$. As $n$ ranges from zero to larger and larger values, the enhancement factor ranges from one to ever larger values (in agreement, let us say, with a principle that we call inclusion principle). The fermionic transition rates $r_{12}^{f}, r_{21}^{f}$ can be expressed in terms of the rates for classical particles multiplying the classical rates by $1-n$ inhibition factor; $n$ ranges from zero to one, the inhibition factor ranges from one to zero, in agreement with the exclusion principle. The application of these relations to the detailed balancing postulate allows us to derive the classical Maxwell-Boltzmann (MB) distribution and the quantum FD and BE distributions in a very simple way.

The usual, linear Fokker-Planck (FP) equation describes the time dependent, classical distribution function $n(t, \mathbf{v})$ and the transition probability rates are at the basis of the derivation of this equation. To our knowledge, up to now, no inhibition or enhancement factor has ever been set in the transition probability rates to obtain, directly from the analytical or numerical solution of the FP equation, the distribution functions of fermions and bosons at any time towards equilibrium. The research of quantum statistical distribution and of their evolution in time towards equilibrium from classical kinetic equations is a very stimulating task. These equations should contain particular constraints on the transition probability rates from one occupancy site to another one. In the case of fermions the problem has been treated by some authors inserting a repulsive potential into the diffusion equations, in such a way that fermions do not collapse to the lowest level [1]. By means of this method one obtains distributions close to the FD one, however a full agreement is not at all reached. Our approach is quite different.

In this work we follow and generalize the approach already explained by us in a previous paper, which was limited to 1-dimensional fermionic particles [2]. At the basis of this work we consider first of all the transition rates and, taking into account the quantum effects deriving from the indistinguishability of identical particles, we give an expression of them by means of a factor which contains, fully or partially, the inhibition or the enhancement 
effect. The graduality of the inhibition or the enhancement is assured by a parameter $\kappa$ whose values range between -1 and +1 . Then, as a consequence, we derive a non-linear kinetic equation. In some case, like in the brownian particle case, analytical solutions at the equilibrium, as time goes to infinite, can be deduced in a simple way; in other cases one could derive numerical solutions for the time dependent distribution functions. As we shall see, we may obtain also statistical distributions different from the FD and BE ones, when the parameter $\kappa$ contained in the inhibition or enhancement factor has values between -1 and +1 , except the value zero.

In the last years a great effort has been spent in studying intermediate statistics interpolating between FD and BE statistics. We wish to recall here the early work related to this subject by Leinaas and Mirheim [3]. They have examined the classical configuration space of identical particles and shown that the classical description of a many-particle system is affected by indistinguishability of the particles. The configuration space of a $\mathrm{N}$-particle system is not the Cartesian product of the single particle spaces, but rather an identification space. This fact is used in the quantized theory and has, as a consequence, that no symmetry constraints on the wave function need to be postulated. In fact, since the indistinguishability of the particles is taken into account in the definition of the configuration space, no additional restriction, corresponding to the symmetrization postulate, is put on the state functions. The restriction on wave functions, to be either symmetric or antisymmetric then appears in a natural way from the formalism, without having to be introduced as an additional constraint. This is however only the case if the particles move in a three or higher dimensional space. In one and two dimensions it is shown that also intermediate cases between boson and fermion systems are possible. The non-linear kinetic equations we use are defined in an arbitrary dimensional space. In contrast to the above quantum case, we can deduce, in addition to the FD an the BE distributions, also a family of different distributions, whose existence is also related to the research of small violations of the FD and BE statistics, i.e. of the exclusion Pauli principle and of the inclusion principle.

The research of statistical distributions interpolating between the FD and $\mathrm{BE}$ ones and 
allowing that $N$ fermions, in place of one alone, occupy a single state, started with the early work by Gentile in 1940 [4]. Very recently the search for small violations of the FD and BE statistics has developed many studies on the intermediate statistics, where creation and annihilation operators obey to commutation relations interpolating between bosons and fermions [5-7]. Experimental high precision methods have been developed to verify small deviations from the Pauli principle. Implications of such deviations are reported in atomic, nuclear, particle and condensed matter physics and in astrophysics and cosmology.

Indistinguishability is strictly related to antisymmetrisation and to symmetrisation of wave functions. Let us introduce the concept of degree of antisymmetrisation or degree of symmetrisation. In some physical systems of many-identical fermions trial wave functions are used being characterized by a partial antisymmetrisation: only with this kind of wave functions it is possible to describe the physical properties of systems. One example is the trial wave function studied by Brovetto and Bussetti [8] to analyze the hyperfine structure of the free neutral hydrocarbon aromatic radicals. In nuclear physics the independent boson model (IBM) describes the main properties (i.e. energy levels, electric quadrupole moments) of heavy nuclei. However IBM contains fundamental problems as for instance the bosonization of fermions, the fact that we cannot antisymmetrize completely all the nucleons and that particle-particle pairs of nucleons can be coupled to angular momentum two, although we know that pairs of fermions are not completely bosons, but only approximately so.

Complete antisymmetrisation means complete indistinguishability. We shall introduce the inhibition or enhancement factor $1+\kappa n(t, \mathbf{v})$ into the transition probabilities rates. As a consequence the kinetic equation becomes non-linear because the factor contains the distribution function $n(t, \mathbf{v})$. The values of the parameter $\kappa$ ranges between -1 and +1 , therefore, as we shall see, varying the value of $\kappa$, we can consider both the BE case $\kappa=+1$ and the FD case $\kappa=-1$, in addition to the classical MB case $\kappa=0$ and to all intermediate cases when $\kappa$ assumes one of the intermediate values between -1 and +1 . The parameter $\kappa$ has the meaning of degree of indistinguishability of fermions or of bosons, corresponding to the degree of antisymmetrisation or of symmetrisation, respectively. Depending on the value 
of $\kappa$ we may choose the degree of indistinguishability or the degree of classicity of the system of particles under consideration. The result obtained in this paper holds promise that it may be possible to develop interesting models for atomic, nuclear and particle quantum dynamics within the framework of classical, non-linear equations of motion containing quantum effects through inhibition or enhancement factors. We send to ref.[9] for a classical kinetic model of intermediate statistics.

In Sec.II, we introduce a general, non-linear kinetic equation in an arbitrary Ddimensional space that, in the case of $\kappa=0$, becomes the well-known, linear Fokker-Planck equation.

In Sec.III, we limit ourselves to consider nearest neighbors interactions and only first and second order momentum of the transition rate different from zero. A stationary distribution is derived whose expression is valid for any value of the parameter $\kappa$, between -1 and +1 .

In Sec.IV, we develop the kinetics for Brownian particles: the drift coefficient is proportional to the particle velocity and the diffusion coefficient is a constant. As the time goes to infinite we may derive the expression of the distribution function which coincides, when $\kappa= \pm 1$, to the $\mathrm{BE}$ and $\mathrm{FD}$ distribution and when $\kappa=0$ to the $\mathrm{MB}$ one.

In Sec.V, the kinetics of the previous section is studied on a lattice i.e. on a discrete velocity (or discrete energy) space. We may deduce the quantum of energy which is the interval between two close states. The distribution functions are also derived.

Conclusions are reported in Sec.VI.

\section{PARTICLE KINETICS IN THE PHASE SPACE}

Let us consider the kinetics of particles in a continuous space of arbitrary dimension $D$. We call $n(t, \mathrm{v})$ the occupational number, it is a scalar field which describes univocally the particle distribution at any point of the phase space. The particle kinetics is described by the transition probability $\pi(t, \mathbf{v} \rightarrow \mathbf{u})$ from the point $\mathbf{v}$ to the point $\mathbf{u}$ in the phase space.

The evolution equation can be written in the form: 


$$
\frac{\partial n(t, \mathbf{v})}{\partial t}=\int[\pi(t, \mathbf{u} \rightarrow \mathbf{v})-\pi(t, \mathbf{v} \rightarrow \mathbf{u})] d^{D} u
$$

The variation in time of $n(t, \mathrm{v})$ is due exclusively to the difference between the particles arriving in $\mathbf{v}$ from any point of the space and the particles leaving $\mathbf{v}$ and going towards any point of the space.

Let us define the transition probability of a particle from the state $\mathbf{v}$ to the state $\mathbf{u}$ in the following way:

$$
\pi(t, \mathbf{v} \rightarrow \mathbf{u})=r(t, \mathbf{v}, \mathbf{v}-\mathbf{u}) \varphi[n(t, \mathbf{v})] \psi[n(t, \mathbf{u})]
$$

where $r(t, \mathbf{v}, \mathbf{v}-\mathbf{u})$ is the transition rate, depending on the initial state $\mathbf{v}$ and on the variation $\mathbf{v}-\mathbf{u}$ of the particle state during the transition, $\varphi$ and $\psi$ are functions of the occupational number to be fully expressed: $\varphi[n(t, \mathbf{v})]$ is a function depending on the occupational distribution at the initial state $\mathbf{v}$ while $\psi[n(t, \mathbf{u})]$ depends on the occupational distribution of the arrival site $\mathbf{u}$. These two functions can inhibit or enhance, fully or partially, at a certain degree, the transition rates, from site to site.

The evolution equation, taking into account the expression of the transition probability, assumes the form:

$$
\begin{aligned}
\frac{\partial n(t, \mathbf{v})}{\partial t}= & \psi[n(t, \mathbf{v})] \int r(t, \mathbf{v}+\mathbf{u}, \mathbf{u}) \varphi[n(t, \mathbf{v}+\mathbf{u})] d^{D} u \\
& -\varphi[n(t, \mathbf{v})] \int r(t, \mathbf{v}, \mathbf{u}) \psi[n(t, \mathbf{v}-\mathbf{u})] d^{D} u
\end{aligned}
$$

In this paper we consider physical systems evolving very slowly. This means that in the first integral of the right hand side of Eq.(3) the quantity $r(t, \mathbf{v}+\mathbf{u}, \mathbf{u}) \varphi[n(t, \mathbf{v}+\mathbf{u})]$ can be expanded in a Taylor series as a function of $\mathbf{v}+\mathbf{u}$, in an interval around $\mathbf{v}$, being $u \ll v$. We obtain:

$$
r(t, \mathbf{v}+\mathbf{u}, \mathbf{u}) \varphi[n(t, \mathbf{v}+\mathbf{u})]=\sum_{m=0}^{\infty} \frac{1}{m !} \frac{\partial^{m}\{r(t, \mathbf{v}, \mathbf{u}) \varphi[n(t, \mathbf{v})]\}}{\partial v_{\alpha_{1}} \partial v_{\alpha_{2}} \ldots \partial v_{\alpha_{m}}} u_{\alpha_{1}} u_{\alpha_{2}} \ldots u_{\alpha_{m}}
$$

The function $\psi[n(t, \mathbf{v}-\mathbf{u})]$ also, in the second integral of Eq.(3), can be written as:

$$
\psi[n(t, \mathbf{v}-\mathbf{u})]=\sum_{m=0}^{\infty} \frac{(-1)^{m}}{m !} \frac{\partial^{m} \psi[n(t, \mathbf{v})]}{\partial v_{\alpha_{1}} \partial v_{\alpha_{2}} \ldots \partial v_{\alpha_{m}}} u_{\alpha_{1}} u_{\alpha_{2}} \ldots u_{\alpha_{m}}
$$


After the introduction of the $m^{\text {th }}$ order momentum of the transition rate

$$
\zeta_{\alpha_{1} \alpha_{2} \ldots \alpha_{m}}(t, \mathbf{v})=\frac{1}{m !} \int r(t, \mathbf{v}, \mathbf{u}) u_{\alpha_{1}} u_{\alpha_{2}} \ldots u_{\alpha_{m}} d^{D} u
$$

the integro-differential Eq.(3) can be transformed into the following infinite order differential equation:

$$
\begin{aligned}
\frac{\partial n(t, \mathbf{v})}{\partial t}= & \sum_{m=0}^{\infty}\left(\psi \left[n(t, \mathbf{v}] \frac{\partial^{m}\left\{\zeta_{\alpha_{1} \alpha_{2} \ldots \alpha_{m}}(t, \mathbf{v}) \varphi[n(t, \mathbf{v})]\right\}}{\partial v_{\alpha_{1}} \partial v_{\alpha_{2}} \ldots \partial v_{\alpha_{m}}}\right.\right. \\
& \left.+(-1)^{m+1} \zeta_{\alpha_{1} \alpha_{2} \ldots \alpha_{m}}(t, \mathbf{v}) \varphi[n(t, \mathbf{v})] \frac{\partial^{m} \psi[n(t, \mathbf{v})]}{\partial v_{\alpha_{1}} \partial v_{\alpha_{2}} \ldots \partial v_{\alpha_{m}}}\right)
\end{aligned}
$$

We observe that the functions $\varphi(n)$ and $\psi(n)$ must obey the two conditions, in the case the initial or arrival site is empty:

$$
\varphi(0)=0, \quad \psi(0)=1
$$

The first condition means we do not have transitions when the initial site is empty, while the second condition imposes that the transition probability does not depend on the arrival site if, in it, particles are absent. If, on the contrary, the arrival site is populated, we can impose that its population affects the transition probability. In the more simple case where $\varphi(n)$ and $\psi(n)$ are linear functions of $n$, we have:

$$
\varphi(n)=n, \quad \psi(n)=1+\kappa n,
$$

where $\kappa$ is a parameter which has the value -1 in case the particles are fermions and +1 in case are bosons. In the first case, $\psi(n)$ must represent an inhibition factor: if in the arrival site $n=1$, the inhibition factor is zero and transitions do not take place; on the other hand, if $n=0$, we have $\psi(n)=1$ and transitions shall take place. In the case particles are bosons $\kappa$ is equal to 1 and the probability transition is ever larger as the occupational number $n$ in the arrival site increases. The relation $\varphi(n)=n$ means that if the initial state contains a high number of particles, the probability that one of them leaves the state increases linearly with the occupation number of the starting site. Taking into account Eq.s (9), the evolution equation given by Eq.(7) becomes: 


$$
\begin{gathered}
\frac{\partial n(t, \mathbf{v})}{\partial t}=\sum_{m=0}^{\infty} \frac{\partial^{m}\left[\zeta_{\alpha_{1} \alpha_{2} \ldots \alpha_{m}}(t, \mathbf{v}) n(t, \mathbf{v})\right]}{\partial v_{\alpha_{1}} \partial v_{\alpha_{2}} \ldots \partial v_{\alpha_{m}}} \\
+\kappa n(t, \mathbf{v}) \sum_{m=0}^{\infty}\left[\frac{\partial^{m}\left[\zeta_{\alpha_{1} \alpha_{2} \ldots \alpha_{m}}(t, \mathbf{v}) n(t, \mathbf{v})\right]}{\partial v_{\alpha_{1}} \partial v_{\alpha_{2}} \ldots \partial v_{\alpha_{m}}}+(-1)^{m+1} \zeta_{\alpha_{1} \alpha_{2} \ldots \alpha_{m}}(t, \mathbf{v}) \frac{\partial^{m} n(t, \mathbf{v})}{\partial v_{\alpha_{1}} \partial v_{\alpha_{2}} \ldots \partial v_{\alpha_{m}}}\right]
\end{gathered}
$$

which describes a non-linear kinetics. We note that the parameter $\kappa$ gives a quantification of how much the particle kinetics is affected by the particle population of the arrival site. In the case $\kappa=0$ we obtain the well known, linear Fokker-Planck equation.

\section{NEAREST NEIGHBOURS INTERACTION}

In this section we consider the Eq.(10) in the case only the first and the second order momentum of the transition rate are different from zero; we have:

$$
\begin{gathered}
\zeta_{\alpha}(t, \mathbf{v})=\int r(t, \mathbf{v}, \mathbf{u}) u_{\alpha} d^{D} u \\
\zeta_{\alpha \beta}(t, \mathbf{v})=\frac{1}{2} \int r(t, \mathbf{v}, \mathbf{u}) u_{\alpha} u_{\beta} d^{D} u .
\end{gathered}
$$

Eq.(10) can be written as it follows:

$$
\frac{\partial n(t, \mathbf{v})}{\partial t}=\frac{\partial}{\partial v_{\alpha}}\left\{\left[\zeta_{\alpha}(t, \mathbf{v})+\frac{\partial \zeta_{\alpha \beta}(t, \mathbf{v})}{\partial v_{\beta}}\right] n(t, \mathbf{v})[1+\kappa n(t, \mathbf{v})]+\zeta_{\alpha \beta}(t, \mathbf{v}) \frac{\partial n(t, \mathbf{v})}{\partial v_{\beta}}\right\} .
$$

We define the particle current $j_{\alpha}(t, \mathbf{v})$ by means of the relation:

$$
j_{\alpha}(t, \mathbf{v})=-\left[\zeta_{\alpha}(t, \mathbf{v})+\frac{\partial \zeta_{\alpha \beta}(t, \mathbf{v})}{\partial v_{\beta}}\right] n(t, \mathbf{v})[1+\kappa n(t, \mathbf{v})]-\zeta_{\alpha \beta}(t, \mathbf{v}) \frac{\partial n(t, \mathbf{v})}{\partial v_{\beta}}
$$

The last term on the right hand side of Eq.(14) is the Fick current, while the first term is a drift current depending on $\zeta_{\alpha}(t, \mathbf{v}), \partial \zeta_{\alpha \beta}(t, \mathbf{v}) / \partial v_{\beta}$ and on the parameter $\kappa$ related to the exclusion-inclusion principle. After introducing the particle current given by Eq.(14), Eq.(13) has the expression:

$$
\frac{\partial n(t, \mathbf{v})}{\partial t}+\nabla \mathbf{j}(t, \mathbf{v})=0
$$

which is the continuity equation and takes into account the local balance in the phase space. 
We demonstrate that the kinetics described by Eq.(15) takes into account only interactions among the nearest neighbors. If we make the hypothesis that during a single transition only one component of the position vector $\mathbf{u}$ can change of a quantity $\Delta v$, the transition rate assumes the following form:

$$
\begin{aligned}
r(t, \mathbf{v}, \mathbf{u})=\frac{1}{\Delta v^{2}} \sum_{\alpha=1}^{D} \prod_{\beta \neq \alpha} & \delta\left(u_{\beta}\right)\left\{\left[D_{\alpha}(t, \mathbf{v})+\frac{1}{2} J_{\alpha}(t, \mathbf{v}) \Delta v\right] \delta\left(u_{\alpha}-\Delta v\right)\right. \\
+ & {\left.\left[D_{\alpha}(t, \mathbf{v})-\frac{1}{2} J_{\alpha}(t, \mathbf{v}) \Delta v\right] \delta\left(u_{\alpha}+\Delta v\right)\right\} }
\end{aligned}
$$

where $D_{\alpha}(t, \mathbf{v})$ and $J_{\alpha}(t, \mathbf{v})$ are the diffusion and the drift coefficients. With this choice of the transition rate and using Eq.(6) we have, for $\Delta v \rightarrow 0$ the following expressions for the momenta:

$$
\begin{aligned}
\zeta_{\alpha}(t, \mathbf{v}) & =J_{\alpha}(t, \mathbf{v}), \\
\zeta_{\alpha \beta}(t, \mathbf{v}) & =D_{\alpha}(t, \mathbf{v}) \delta_{\alpha \beta}, \\
\zeta_{\alpha_{1} \alpha_{2} \ldots \alpha_{m}}(t, \mathbf{v}) & =0 \quad m>2 .
\end{aligned}
$$

Eq.(17) represents the conditions to be satisfied only for the nearest neighbors interaction. Let us remark that the second order momentum is a diagonal matrix. This fact has the consequence that during the transitions the various components of the current vector do not mix with each other; we have:

$$
j_{\alpha}(t, \mathbf{v})=-\left[J_{\alpha}(t, \mathbf{v})+\frac{\partial D_{\alpha}(t, \mathbf{v})}{\partial v_{\alpha}}\right] n(t, \mathbf{v})[1+\kappa n(t, \mathbf{v})]-D_{\alpha}(t, \mathbf{v}) \frac{\partial n(t, \mathbf{v})}{\partial v_{\alpha}}
$$

In Eq. (18) repeated indices are not summed over. Substituting this expression of the current into Eq.(10) we obtain:

$$
\frac{\partial n(t, \mathbf{v})}{\partial t}=\nabla\left\{\left[\mathbf{J}(t, \mathbf{v})+\frac{\partial D_{\alpha}(t, \mathbf{v})}{\partial v_{\alpha}} \hat{\alpha}\right] n(t, \mathbf{v})[1+\kappa n(t, \mathbf{v})]+D_{\alpha}(t, \mathbf{v}) \frac{\partial n(t, \mathbf{v})}{\partial v_{\alpha}} \hat{\alpha}\right\}
$$

that is exactly the Eq.(15). This equation can be obtained also directly, by means of a local balance of the particles along the generic direction $\alpha$. Let us consider an infinitesimal variation $d \mathrm{v}$ of the position of a particle along the direction $\alpha$; we have: 


$$
(d \mathbf{v})_{\beta}=\delta_{\beta \alpha} d v
$$

Defining the transition rate by the relation:

$$
r(t, \mathbf{v}, \pm d \mathbf{v})=D_{\alpha}(t, \mathbf{v}) \pm \frac{1}{2} J_{\alpha}(t, \mathbf{v}) d v
$$

the transition probabilities of the two transitions $\mathbf{v} \rightarrow \mathbf{v}+d \mathbf{v}$ and $\mathbf{v}+d \mathbf{v} \rightarrow \mathbf{v}$ assume the following expressions:

$$
\begin{gathered}
\pi(t, \mathbf{v} \rightarrow \mathbf{v}+d \mathbf{v})=r(t, \mathbf{v}, d \mathbf{v}) n(t, \mathbf{v})[1+\kappa n(t, \mathbf{v}+d \mathbf{v})] \\
\pi(t, \mathbf{v}+d \mathbf{v} \rightarrow \mathbf{v})=r(t, \mathbf{v}+d \mathbf{v},-d \mathbf{v}) n(t, \mathbf{v}+d \mathbf{v})[1+\kappa n(t, \mathbf{v})]
\end{gathered}
$$

The particle current along the direction $\alpha$ is consequently given by:

$$
j_{\alpha}(t, \mathbf{v}) d v=\pi(t, \mathbf{v} \rightarrow \mathbf{v}+d \mathbf{v})-\pi(t, \mathbf{v}+d \mathbf{v} \rightarrow \mathbf{v})
$$

and explicitly we have:

$j_{\alpha}(t, \mathbf{v}) d v=r(t, \mathbf{v}, d \mathbf{v}) n(t, \mathbf{v})[1+\kappa n(t, \mathbf{v}+d \mathbf{v})]-r(t, \mathbf{v}+d \mathbf{v},-d \mathbf{v}) n(t, \mathbf{v}+d \mathbf{v})[1+\kappa n(t, \mathbf{v})]$.

If we expand this expression, up to the first order in $d v$, we obtain immediately Eq.(18) and consequently Eq.(19).

We pose now our attention to the steady states of Eq.(19). We define:

$$
\begin{aligned}
J_{\alpha}(\mathbf{v}) & =\lim _{t \rightarrow \infty} J_{\alpha}(t, \mathbf{v}) \\
D_{\alpha}(\mathbf{v}) & =\lim _{t \rightarrow \infty} D_{\alpha}(t, \mathbf{v}), \\
n(\mathbf{v}) & =\lim _{t \rightarrow \infty} n(t, \mathbf{v}) .
\end{aligned}
$$

The stationary solutions $n(\mathbf{v})$ can be obtained directly by observing that at the limit of $t \rightarrow \infty$ the particle current is equal to zero. It is easy to integrate the first order differential equation $\mathbf{j}(t, \mathbf{v})=0$, when $J_{\alpha}(\mathbf{v})$ and $D_{\alpha}(\mathbf{v})$ are related by means of the following expression: 


$$
J_{\alpha}(\mathbf{v})=h_{\alpha}\left(v_{\alpha}\right) D_{\alpha}(\mathbf{v})-\frac{\partial D_{\alpha}(\mathbf{v})}{\partial v_{\alpha}}
$$

where $h_{\alpha}\left(v_{\alpha}\right)$ is an arbitrary function. We obtain with easy calculations:

$$
n(\mathrm{v})=\frac{1}{\exp \left[\sum_{\alpha=1}^{N} \int h_{\alpha}\left(v_{\alpha}\right) d v_{\alpha}-\beta \mu\right]-\kappa},
$$

where $\beta \mu$ is the integration constant and can be calculated from the normalization condition that expresses the conservation of the particle number.

The condition given by Eq.(29) is satisfied if the motion along the various directions of the phase space is completely decoupled:

$$
\begin{gathered}
J_{\alpha}(\mathbf{v})=J_{\alpha}\left(v_{\alpha}\right) \\
D_{\alpha}(\mathbf{v})=D_{\alpha}\left(v_{\alpha}\right)
\end{gathered}
$$

In this case the steady state given by Eq.(30) assumes the expression:

$$
n(\mathbf{v})=\frac{1}{\exp \left[\sum_{\alpha=1}^{N} \int \frac{1}{D_{\alpha}\left(v_{\alpha}\right)}\left[J_{\alpha}\left(v_{\alpha}\right)+\frac{\partial D_{\alpha}\left(v_{\alpha}\right)}{\partial v_{\alpha}}\right] d v_{\alpha}-\beta \mu\right]-\kappa} .
$$

Eq.(33) is the most general expression for the stationary distribution function $n(v)$ when the motion along the various directions is decoupled. Depending on the expressions of $J_{\alpha}\left(v_{\alpha}\right)$ and $D_{\alpha}\left(v_{\alpha}\right)$ the integration can be analytically or numerically accomplished. The parameter $\kappa$ can vary continuously between -1 and +1 : all kind of statistics can be derived, in the framework of validity of the nearest neighbors interaction hypothesis.

\section{BOSONS AND FERMIONS IN A CONTINUOUS SPACE}

In this Section we consider the brownian particle kinetics in a 3-dimensional space; for this type of particles, the drift coefficient is proportional to the particle velocity while the diffusion coefficient is a constant: 


$$
J_{\alpha}(t, \mathbf{v})=c v_{\alpha}, \quad D_{\alpha}(t, \mathbf{v})=\frac{c}{\beta m}, \quad \beta=\frac{1}{k T}
$$

where $c$ is an arbitrary constant. The current is given by:

$$
\mathbf{j}=-c \mathbf{v} n(t, \mathbf{v})[1+\kappa n(t, \mathbf{v})]-\frac{c}{\beta m} \nabla n(t, \mathbf{v})
$$

The kinetics equation assumes the form:

$$
\frac{\partial n(t, \mathbf{v})}{\partial t}=\nabla\left\{c \mathbf{v} n(t, \mathbf{v})[1+\kappa n(t, \mathbf{v})]+c \frac{1}{\beta m} \nabla n(t, \mathbf{v})\right\}
$$

Let us remark that Eq.(36) is a continuity equation, written in the velocity space. Later on we will show that Eq.(36) can be written as a continuity equation in the energy variable. The kinetic energy of a particle is given by:

$$
E=\frac{1}{2} m\left(v_{1}^{2}+v_{2}^{2}+v_{3}^{2}\right)
$$

We now indicate with $N(t, v)$ the number of particles having at time $t$ the modulus of velocity $\leq v$. Of course these are the particles lying, in the velocity space, in a sphere $\Sigma_{v}$ having the center at the origin and the radius equal to $v$; we have:

$$
N(t, v)=\int_{\Sigma_{v}} n(t, \mathbf{v}) d^{3} v
$$

Let us call $N$ the total number of particles. This number must be conserved at any time and therefore we have the condition:

$$
N(t, \infty)=N
$$

If we indicate with $\Phi(t, v)$ the flux of particles outcoming the sphere $\Sigma_{v}$ at time $t$ we have:

$$
\Phi(t, v)=\int_{\Sigma_{\mathbf{v}}} \mathbf{j}(t, \mathbf{v}) \mathbf{n} d \sigma
$$

the equation describing the evolution of the particles inside the sphere $\Sigma_{v}$ is:

$$
\frac{\partial N(t, v)}{\partial t}+\Phi(t, v)=0
$$


We differentiate Eq.(41) with respect to $v$ and take into account Eq.(38); we can write Eq.(41) in the form:

$$
\frac{\partial n(t, \mathbf{v})}{\partial t}+\frac{1}{4 \pi v^{2}} \frac{\partial \Phi(t, v)}{\partial v}=0
$$

The values of $\Phi(t, v)$ can easily be calculated by Eq.(40) which assumes the expression:

$$
\Phi(t, v)=4 \pi v \mathbf{v} \mathbf{j}(t, \mathbf{v})
$$

Then Eq.(42) assumes the following form:

$$
\frac{\partial n(t, \mathbf{v})}{\partial t}=\frac{c}{v^{2}} \frac{\partial}{\partial v}\left(v^{2}\left\{v n(t, \mathbf{v})[1+\kappa n(t, \mathbf{v})]+\frac{1}{\beta m} \frac{\partial n(t, \mathbf{v})}{\partial v}\right\}\right)
$$

and, after the introduction of the energy as the variable, we have:

$$
\frac{\partial n(t, E)}{\partial t}=\frac{2 c}{E^{1 / 2}} \frac{\partial}{\partial E}\left(E^{3 / 2}\left\{n(t, E)[1+\kappa n(t, E)]+\frac{1}{\beta} \frac{\partial n(t, E)}{\partial E}\right\}\right) .
$$

We now want to interpret Eq.(45) as a continuity equation in the one-dimensional energy space: we introduce the occupation probability $p(t, E)$ at energy $E$, then the number of particles that lie in the interval $[E, E+d E]$ is equal to the number of particles lying in the shell delimited by the two spheres $\Sigma_{v+d v}$ and $\Sigma_{v}$, having the modulus of the velocity in the interval $[v, v+d v]$; we therefore have:

$$
p(t, E) d E=4 \pi v^{2} n(t, E) d v
$$

From Eq.(46) it follows that the function $p(t, E)$ is given by:

$$
p(t, E)=2 \pi\left(\frac{2}{m}\right)^{3 / 2} \sqrt{E} n(t, E)
$$

We introduce the constant:

$$
a=4 c \pi\left(\frac{2}{m}\right)^{3 / 2}
$$

The flux of particles $\Phi(t, E)$ outcoming the sphere $\Sigma_{v}$ of radius $v=\sqrt{2 E / m}$ represents, of course, the particle current in the energy axis and assumes the following form: 


$$
\Phi(t, E)=-a E^{3 / 2}\left\{n(t, E)[1+\kappa n(t, E)]+\frac{1}{\beta} \frac{\partial n(t, E)}{\partial E}\right\}
$$

Eq.(45) assumes finally the form of a continuity equation in the one-dimensional energy space:

$$
\frac{\partial p(t, E)}{\partial t}+\frac{\partial \Phi(t, E)}{\partial E}=0
$$

If we set the particle current $\Phi(t, E)$ equal to zero we obtain that the stationary distribution at the limit $t \rightarrow \infty$ must satisfy by the first order differential equation:

$$
n(E)[1+\kappa n(E)]+\frac{1}{\beta} \frac{\partial n(E)}{\partial E}=0
$$

After integration we have:

$$
n(E)=\frac{1}{\exp \beta(E-\mu)-\kappa} .
$$

The integration constant $\mu$ can be determined from the normalization condition:

$$
\int n(E) d \tau=N
$$

where $d \tau$ is the elementary volume in the phase space and is given by $d \tau=d \tau_{1} d \tau_{2} d \tau_{3}$ with $d \tau_{i}=d\left(m v_{i}\right) d x_{i} / h . N$ is the total number of particles of the system occupying the volume $V$ and $h$ is a constant with the right dimensions which can be identified by the Planck constant. After the introduction of the constant $\mathcal{N}$, defined by:

$$
\mathcal{N}=\frac{\rho}{2 \pi}\left(\frac{\beta h^{2}}{2 m}\right)^{3 / 2}
$$

the condition given by Eq.(53) assumes the form:

$$
\int_{-\beta_{\mu}}^{\infty} \frac{\sqrt{z+\beta \mu}}{\exp z-\kappa} d z=\mathcal{N}
$$

Eq. (52) gives exactly the FD $(\kappa=-1), \mathrm{MB}(\kappa=0)$ and $\mathrm{BE}(\kappa=+1)$ statistics and for all the other values of $\kappa$ a family of different statistical distributions. 


\section{BOSONS AND FERMIONS IN A LATTICE}

In this section we wish to discuss, in a discrete velocity space, the kinetics of brownian particles. The results for this case can be useful when energies are discretized and the interval between close levels is represented by a quantum of energy. Let us assume that only transitions to the nearest neighbors are allowed and that the direction of the particles does not change during the transition. The Pauli master equation for this process can be written in the following way:

$$
\frac{d n_{i}(t)}{d t}=\pi\left(t, v_{i-1} \rightarrow v_{i}\right)+\pi\left(t, v_{i+1} \rightarrow v_{i}\right)-\pi\left(t, v_{i} \rightarrow v_{i+1}\right)-\pi\left(t, v_{i} \rightarrow v_{i-1}\right)
$$

where $\pi\left(t, v_{i} \rightarrow v_{j}\right)$ is the transition probability from the site $i$ to the site $j$ and $n_{i}(t)$ is the occupation probability of the site $i$. We define the particle current as:

$$
j_{i}(t)=\left[\pi\left(t, v_{i} \rightarrow v_{i+1}\right)-\pi\left(t, v_{i+1} \rightarrow v_{i}\right)\right] \Delta v
$$

and call the current variation between two adjacent sites:

$$
\Delta j_{i}(t)=j_{i}(t)-j_{i-1}(t)
$$

The Pauli master equation given by Eq.(56) assumes an expression analogous to the continuity equation in a discrete space:

$$
\frac{d n_{i}(t)}{d t}+\frac{\Delta j_{i}(t)}{\Delta v}=0
$$

We now introduce the drift $J_{i}(t)$ and the diffusion $D_{i}(t)$ coefficients by means of the transition rates:

$$
\begin{aligned}
J_{i}(t) \Delta v & =r\left(t, v_{i},+\Delta v\right)-r\left(t, v_{i},-\Delta v\right) \\
2 D_{i}(t) & =r\left(t, v_{i+1},-\Delta v\right)+r\left(t, v_{i+1},-\Delta v\right) .
\end{aligned}
$$

We consider the definition of the transition probability of Eq.(2), where the functions $\varphi(n)$ and $\psi(n)$ are given by Eq.(9) and remember that now the velocity is a discrete variable, we can write the expression for the particle current : 


$$
j_{i}(t)=-\left\{\left[J_{i}(t)+\frac{\Delta D_{i}(t)}{\Delta v}\right] n_{i}(t)\left[1+\kappa n_{i+1}(t)\right]+D_{i}(t) \frac{\Delta n_{i}(t)}{\Delta v}\right\} \Delta v .
$$

We are considering brownian motion by imposing that the drift and the diffusion coefficients have the following expressions:

$$
J_{i}(t)=c v_{i}, \quad D_{i}(t)=\frac{c}{m \beta}
$$

Considering the energy as the variable instead of the velocity and taking into account that:

$$
\frac{\Delta n_{i}(t)}{\Delta v}=m v_{i} \frac{\Delta n_{i}(t)}{\Delta E}
$$

the expression of the current assumes the form:

$$
j_{i}(t)=-\frac{c}{m} \Delta E\left\{n_{i}(t)\left[1+\kappa n_{i+1}(t)\right]+\frac{1}{\beta} \frac{\Delta n_{i}(t)}{\Delta E}\right\}
$$

To obtain the stationary distribution of the system, it is enough to pose the particle current equal to zero:

$$
n_{i}\left(1+\kappa n_{i+1}\right)+\frac{n_{i+1}-n_{i}}{\beta \Delta E}=0
$$

In the limit to the continuum we can transform this recurrence relation into the differential equation given by Eq.(51). On the other hand, when we discretize Eq.(51) we must substitute $n(E)(1+\kappa n(E))$ with the quantity $n_{i}\left(1+\kappa n_{i+1}\right)$. It is straightforward to justify this substitution if we remember the definition of the transition probability given by Eq.(2). To deduce $n_{i}$, let us write the recurrence relation of Eq.(66) in the form:

$$
n_{i+1}=\frac{(1-\beta \Delta E) n_{i}}{1+\beta \Delta E \kappa n_{i}}
$$

Introducing the variable $q_{i}$ :

$$
n_{i}=\frac{1}{q_{i}-\kappa}
$$

Eq.(67) become:

$$
q_{i+1}=\frac{1}{1-\beta \Delta E} q_{i}
$$


We can easily calculate $q_{i}$ and then finally $n_{i}$ :

$$
n_{i}=\frac{1}{\exp \beta\left(E_{i}-\mu\right)-\kappa}
$$

which is the discretized distribution. In Eq.(70) the energy $E_{i}$ is given by:

$$
E_{i}=i \mathcal{E}
$$

and $\mathcal{E}$ has the expression:

$$
\mathcal{E}=\frac{1}{\beta} \log \frac{1}{1-\beta \Delta E}
$$

Let us remark that the quantum of energy $\mathcal{E}$, which represents the energy interval between two close states, is different from the lattice interval $\Delta E$. This is due to the finite temperature of the system. Let us expand the energy $\mathcal{E}$ in power series of $\Delta E$, we have:

$$
\mathcal{E} \approx \Delta E+\frac{1}{2} \beta(\Delta E)^{2}+\frac{1}{3} \beta^{2}(\Delta E)^{3}+\ldots
$$

In conclusion the quantum $\mathcal{E}$ coincides with the lattice interval $\Delta E$ only when $\beta \rightarrow 0(T \rightarrow$ $\infty$ ). If we assume that $\mathcal{E}$ is proportional to $\omega$ through the relation $\mathcal{E}=\hbar \omega$, from Eq.(72) we may derive that the relation between $\omega$ and $T$ is given by $\omega=(k T / \hbar) \log [1 /(1-\Delta E / k T)]$, where $k$ is the Boltzmann constant.

Once we consider relation (72), the Eq.(66) can be written in the following form:

$$
n_{i}\left(1+\kappa n_{i+1}\right)=\exp \left[-\beta\left(E_{i+1}-E_{i}\right)\right] n_{i+1}\left(1+\kappa n_{i}\right)
$$

which express the detailed balance principle and is valid for any value of $\kappa \in[-1,+1]$.

Let us remark that if we calculate the limit to the continuum and consider that

$$
\lim _{\Delta E \rightarrow 0} i \Delta E=E
$$

the stationary distribution given by Eq.(70) becomes the continuous distribution given by Eq.(59):

$$
\lim _{\Delta E \rightarrow 0} n_{i}=\frac{1}{\exp \beta(E-\mu)-\kappa} .
$$




\section{CONCLUSIONS}

In this work we introduce a non-linear classical kinetic equation, containing quantum effects introduced into the transition probabilities by means of an inhibition or an enhancement factor. The factor contains a parameter $\kappa$ whose value ranges continuously from -1 and 1 . We take fully into account the exclusion principle when $\kappa=-1$, the inclusion principle when $\kappa=+1$, while the case $\kappa=0$ means that the distribution is a classical one because quantum effects are not considered, in all the other cases different statistical distributions are derived in which the exclusion or the inclusion principle is only partially taken into account, i.e. the particle wave functions are only partially antisymmetrised or symmetrised.

The exact validity of $\mathrm{BE}$ and FD statistics was questioned recently in many papers by Greenberg and collaborators $[6,7]$ in view of considering the possibility of small violations of the Pauli exclusion principle and of BE statistics.

The statistics which differ in a discrete way from the usual ones were introduced by Green and by other authors in the past [5]. Their interest was mainly devoted to the study of transitions caused by a small violation of the Pauli principle rather than to the study of distribution functions of many-particle systems which is the main interest in this work. When the particles are brownian we find exactly the $\mathrm{FD}(\kappa=-1)$, the $\mathrm{BE}(\kappa=1)$ and the classical MB ( $\kappa=0$, no quantum effects are considered). When $\kappa \neq 0, \pm 1$ we have a family of different statistics.

We give to $\kappa$ the meaning of degree of indistinguishability or of degree of antisymmetrisation or symmetrisation, therefore these intermediate statistics can be applied to systems whose wave functions must not be completely antisymmetrised or to physical processes with a small violation of the exclusion or inclusion principle.

The results obtained in this work should not come as a surprise; in fact we know that quantum dynamics is equivalent to classical dynamics with the inclusion of quantum fluctuations. We have limited ourselves in considering explicitly only systems of brownian particles; we must recall that in recent works [10] it has been shown that the quantum average fluc- 
tuations of the quantum dynamics coincides with the brownian fluctuations. Therefore we conclude that the non-linear kinetic equation we have introduced in this paper can describe a dynamics very close to the quantum dynamics of a system of identical particles.

\section{ACKNOWLEDGMENTS}

We thank A. Erdas for critical reading of the manuscript. 


\section{REFERENCES}

[1] C.Dorso, S.Duarte, J.Randrup, Phys.Lett.B 287,1988 (1987).

[2] G.Kaniadakis and P.Quarati, Phys.Rev.E 48,4263 (1993).

[3] J.M.Leinaas,J.Myrheim, Nuovo Cimento 37 B, 1 (1977).

[4] G.Gentile, Nuovo Cimento 17,493 (1940).

[5] H.S.Green, Phys.Rev. 90, 270 (1953).

[6] O.W.Greenberg, R.N.Mohapatra, Phys.Rev.D 39, 2032 (1989).

[7] O.W.Greenberg, Phys.Rev.D 43, 4111 (1991).

[8] P.Brovetto and G.Bussetti, Nuovo Cimento 64 B, 284 (1969).

[9] G.Kaniadakis, Classical intermediate statistics, submitted for publication to Phys.Rev.E together with the present work.

[10] M.Roncadelli, A.Defendi, I cammini di Feynman, Quaderni di Fisica Teorica, Universita' di Pavia, (1992) and references therein. . 